\title{
Low-cycle fatigue behaviour of an aero-engine disk alloy under non-proportional loading
}

\author{
João Vitor Sahadi Cavalheiro ${ }^{1,1}$, and David Nowell $^{2}$ \\ ${ }^{1}$ Department of Engineering Science, University of Oxford, Parks Road, Oxford OX1 3PJ, UK \\ ${ }^{2}$ Department of Mechanical Engineering, Imperial College London, London SW7 2AZ, UK
}

\begin{abstract}
The quest for higher efficiency and fuel economy has pushed aeroengines to challenging levels. In order to become more efficient, engines must run at higher bypass ratios and temperatures, resulting in extreme operating conditions for their hottest section. Nickel based superalloys have been used for this application for the past 50 years due to high fatigue strength at elevated temperatures. This paper investigates the deformation behaviour and fatigue lives of a powder metallurgy Nickel-based superalloy developed for discs of high-pressure turbines, i.e. the most demanding section of aeroengines. For that six different non-proportional load paths were carefully selected, where five of them present the same degree of load non-proportionality, to explore load path dependency and the effects of nonproportional multi-axial loading on fatigue lives. Results confirm an additional cyclic hardening caused by load non-proportionality and its detrimental effect on fatigue life. Lives for non-proportional tests were around three times shorter than fatigue lives for proportional tests at comparable stress levels.
\end{abstract}

\section{Introduction}

Non-proportional loading is defined as any time varying stress/strain path that changes the orientation of the principal stress/strain axes with respect to the axes of the component [1]. Consequently, dislocations move in different directions, interact with each other and generate an additional cyclic hardening, named non-proportional hardening [2]-[4]. A direct effect of this is a noticeable change in the deformation behaviour of the material and a reduction in fatigue life. This phenomenon is especially important for engineering components classified as critical, such as compressor and turbine discs in aeroengine whose failure may be hazardous to the safety of the aircraft.

Turbofan engines, or bypass engines, are carefully designed to meet governmental regulations and customer's demands for greater thrust, reduced fuel consumption and lower noise levels at subsonic flights. The measure of performance for aeroengines is based on thermal efficiency and is strongly dependent upon, and limited by, the high-temperature strength of the nickel-based superalloys used for its hottest sections, i.e. the turbine system [5], [6].

1 Corresponding author: joao.sahadicavalheiro@eng.ox.ac.uk 
High-pressure turbine (HPT) discs, located immediately downstream of the combustion chamber, are the components experiencing the most extreme and demanding conditions. The main function of HPT discs is to position and retain the turbine blades in their correct positions in the axial and radial directions, and in doing so, transfer their circumferential loads to the high-pressure compressor system. In a typical flight cycle, the gas path in the HPT system can reach temperatures of $1500^{\circ} \mathrm{C}$ and spool speeds of $12,000 \mathrm{rpm}$, resulting in great thermal stresses and centrifugal loads (exerted by the spinning disc mass itself and the blades attached to the disc) [7]. The magnitude and contribution of these stresses varies both geometrically across the component, and in time through the flight cycle, creating a true nonproportional multiaxial stress state.

The concentration of thermal energy from the combustion process and kinetic energy in the rotating parts, represent issues for the structural integrity of the engine [8]. Therefore, in order to mitigate the risk of failure, HPT discs are typically manufactured from cast, forged or powder metallurgy processing of polycrystalline nickel-based superalloys with elevated creep resistance, high fatigue strength, low thermal expansion coefficient and high thermal conductivity, fundamental characteristics to sustain the extreme mechanical and thermal loads.

This paper investigates the multi-axial deformation and fatigue behaviour of a powder metallurgy nickel-based superalloys used for HPT discs of aero-engines, named RR1000, under non-proportional loading conditions. For that an extensive analysis has been performed to identify and quantify the load path dependency and the effects of non-proportional multiaxial loading - including additional cyclic hardening and cross-hardening - on fatigue life.

\section{Experimental work}

The test matrix designed to investigate the multiaxial fatigue behaviour of RR1000 consists of 6 non-proportional tension-torsion load paths. In total 16 fully reversed tests, i.e. without mean stresses $\left(R_{\sigma}=-1, R_{\sigma}=\sigma_{\min } / \sigma_{\max }\right)$, were performed under load control at high stress levels (near the yield limit of the material) and room temperature, avoiding creep effects. An ultra-high stiffness tension-torsion servo-hydraulic test machine was used for all tests. The testing rig contains a rotary actuator providing up to $1000 \mathrm{Nm}$ and an axial actuator capable of $100 \mathrm{kN}$. Both actuators are located at the top of the rig and connected to each other in the axial direction so that only the upper grip of the rig is movable, while the lower one is fixed to the machine frame. Manufactured by Instron Testing Systems, the test rig has an advanced testing environment (Wavematrix) that allows the configuration of complex load paths. Due to their complexity and high-loads involved, tests were run at a maximum frequency of $1 \mathrm{~Hz}$.

A camera setup was used for measuring strain fields and detecting the additional hardening caused by load non-proportionality, instead of clip-on extensometers and resistance strain gauges, due to their limitations. Clip-on extensometers have a fixed gauge length which can lead to significant errors at low strain conditions, and stress concentration associated with dimples required to attach the device. On the other hand, resistance bonded strain gauges quality of measurement dependent on the bond, which may not survive long into the fatigue test. The images collected during the tests were used to compare the different levels of non-proportional hardening seen for each load path.

\subsection{Load paths}

The constrained quantity of specimens available, 16, limited the number of load paths that could be investigated, as it is necessary to repeat each load condition at least a few times to 
confirm the real trend and effects of non-proportional hardening. Therefore, 6 load paths were carefully selected for investigation, considering the rotation factor proposed by Kanazawa [4] to quantify the degree of non-proportionality. This factor is mechanistically based on the amount of slip experienced by critical planes in the material and is defined as the ratio between the minor and major axes of the minimum ellipsoid that circumscribe the load path. Figure 1 illustrates the load path dependency (LPD) factor, $F=b / a$, defining the degree of non-proportionality in a range from zero to one. For fully proportional/in-phase tests, $F=0$, whilst for $90^{\circ}$ out-of-phase tests, $F=1$, representing the maximum degree of nonproportionality.

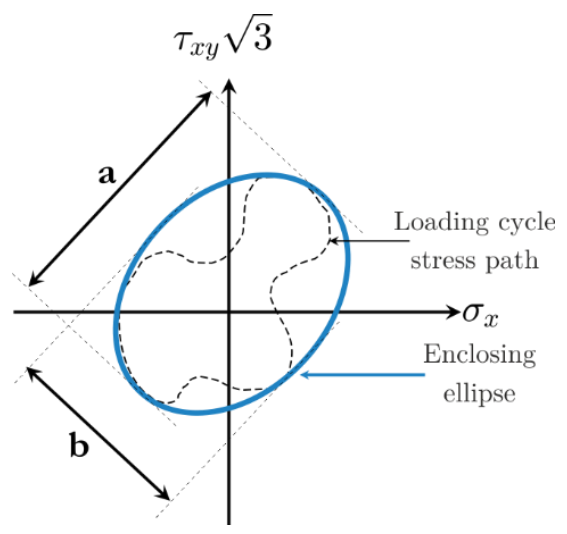

Figure 1 - Load path dependency factor, $F$, adapted from [7].

The first condition tested was the $90^{\circ}$ out-of-phase square load path, named 1SQ, as this is considered the most damaging load path and has been extensively investigated for RR1000 by Bees [2], [8]. The goal was to use Bees' test data as a benchmark to validate the experimental procedure and obtain more data points to accurately predict the nonproportional hardening behaviour of RR1000 under this test condition.

Doong and Socie [9] point out that during out-of-phase loading, the loading sequence and load reversibility have a strong impact on fatigue life. Therefore, in order to investigate the cross-hardening effect caused by sudden changes of loading direction, a modification of the 1SQ load path was proposed. For this proposed load path, named four-square (4SQ), the load is completely removed before each change of direction. Both test conditions present the same LPD factor, $F=1$.

Load cases 1SQ and 4SQ provide enough information for a fundamental analysis of nonproportional hardening and load path dependency. However, they are simplistic load paths that do not consider the real loading conditions that many components experience in service. Therefore, a load path more representative of the stresses seen on HPT disc bore surfaces was selected from the research work presented by Bees [8]. This load path, named two triangles (L1), presents the lowest degree of non-proportionality of all tests, $F=0.44$, a value similar to the LPD factor obtained for non-proportional load paths with a phase difference of $45^{\circ}$.

Analogously to the extension of 1SQ load path to the 4SQ load path, the L1 test condition was adapted for the investigation of cross-hardening. As a result, an hour-glass shaped (HG) load path was obtained, the material thus experiences load reversion in both torsional and axial directions, with $F=1$.

Lastly, two cross load paths (CX1) and (CX2), with $F=1$, were assessed. These two cases present both the opportunity to assess cross-hardening, by contrasting them with the HG load path, and load path dependency, by investigating the effect of load sequence, as this 
is the only difference between the two cases, CX1 and CX2 - CX1 initiates its cycle with a proportional tension-torsion load, whilst CX2 initiates with a proportional compressiontorsion load. Figure 2 illustrate the different load paths investigated in this work and their load sequence, where the red dashed arrow indicates the first loading step.

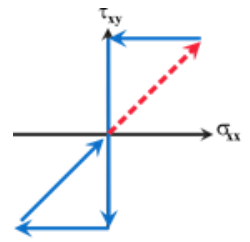

Two Triangle - L1

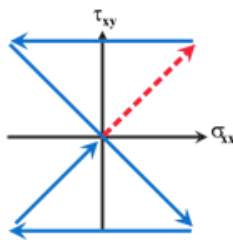

Hour-glass - HG

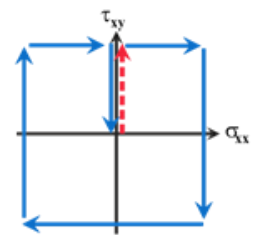

One Square - 1SQ

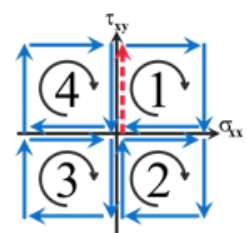

Four Square - 4SQ

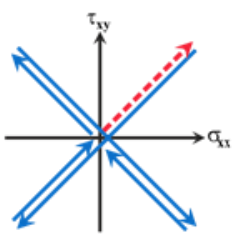

Cross - CX1

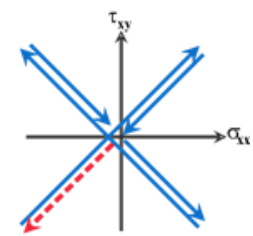

Cross - CX2

Figure 2 - Non-proportional load paths.

\subsection{Specimens}

Thin-walled hollow cylindrical test specimens were used for the stress-controlled fatigue test programme. The geometry of the specimen is presented in Figure 3, where the gauge section has nominal dimensions of $8 \mathrm{~mm}$ and $10 \mathrm{~mm}$ for the inner and outer diameters respectively, and $35 \mathrm{~mm}$ for the gauge length. The specimens were internally reamed and honed, and externally polished across the gauge length to a consistent surface finish. Conical ends to the specimen ensure a uniform circumferential mechanical interface and enable the firm application of torsional loads across a fully reversed load cycle.

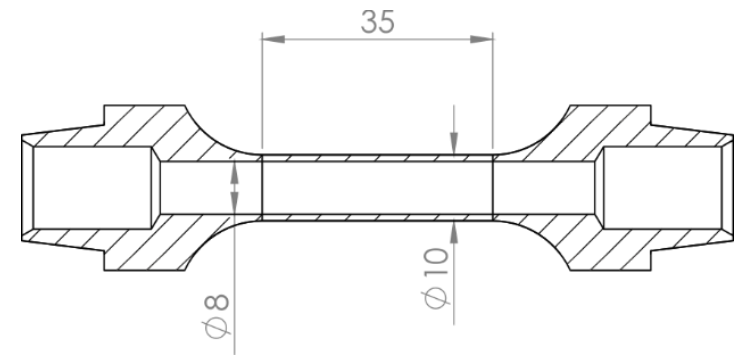

Figure 3 - Geometry of the Tension-Torsion tubular specimen. All dimensions are in $\mathrm{mm}$.

\subsection{Material}

Test specimens were manufactured from RR1000, a fine grain powder-metallurgy nickelbased superalloy developed by Rolls-Royce plc for turbine disc applications. RR1000 specimens were sourced from fully heat-treated and annealed forgings, their chemical 
composition (in wt\%) is given in Table 1. Regarding its mechanical properties, RR1000 presents Young's Modulus, E, of $224100 \mathrm{MPa}$ and Poisson's ratio, $v$, equals to 0.326.

Table 1 - Chemical composition (in wt\%) of RR1000 from [10].

$\begin{array}{cccccccc}\text { Alloy } & \text { Cr } & \text { Co } & \text { Mo } & \text { Al } & \text { Ti } & \text { Ta } & \text { Hf } \\ \text { RR1000 } & 14.35-15.15 & 14.0-19.0 & 4.25-5.25 & 2.85-3.15 & 3.45-4.15 & 1.35-2.15 & 0.0-1.0\end{array}$

\subsection{Stress-strain Formulae}

Static yield parameters characterize the general multiaxial stress state through a scalar quantity, allowing the comparison to simple uniaxial test data. Thus, in order to set the test conditions and correlate the tension-torsion stress state to the uniaxial deformation behaviour of RR1000, the von Mises equivalent stress and strain were implemented. For the tensiontorsion stress condition with only $\sigma_{y} \neq 0$ and $\tau_{x y} \neq 0$, von Mises equivalent stress reduces to

$$
\sigma_{\mathrm{vM}}=\sqrt{\sigma_{y y}^{2}+3 \tau_{x y}^{2}}
$$

and the effective strain can be simplified to

$$
\varepsilon_{\mathrm{eff}}=\sqrt{\varepsilon_{y y}^{2}+\frac{1}{3} \gamma_{x y}^{2}} .
$$

For an idealised elastic/perfectly plastic material, the shear stress is uniform throughout the tubular specimen wall once yield has been exceed at the bore of the tube [11]. Equation (3) gives the average shear stress across the specimen wall thickness,

$$
\tau_{x y, 0}=\frac{3 T}{2 \pi\left(r_{o}^{3}-r_{i}^{3}\right)},
$$

where $T$ is the torque applied and $r_{o}$ and $r_{i}$ are the outer and inner radii respectively. The normal stress, $\sigma_{y y}$, is calculate as follows

$$
\sigma_{y y}=\frac{F}{\pi\left(r_{o}^{2}-r_{i}^{2}\right)}
$$

where $F$ is the axial load applied to the specimen.

Loads for all test conditions were set considering nominal values of von Mises stress, $\sigma_{\mathrm{vM}}$. The normal and shear stresses where obtained using the relationships

$$
\sigma_{y y}=\frac{\sigma_{\mathrm{vM}}}{\sqrt{2}}, \text { and } \tau_{x y, 0}=\frac{\sigma_{y y}}{\sqrt{3}} .
$$

Nominal loads for each test condition were calculated substituting in Eqs. (3) and (4) the results from Eq.(5) for each von Mises stress, $\sigma_{\mathrm{vM}}$, level.

\section{Results}

Table 2 presents the normalised normal, shear and von Mises stress amplitudes. These were calculated based on Eqs (3) and (4), and the axial force and torque measured by the test machine load cell throughout the test. von Mises stress amplitudes were calculated using the formula presented in Eq. (1). 
Figure 4 shows the cyclic stress-strain response for RR1000 with the proportional/inphase (IP) and $90^{\circ}$ out-of-phase (1SQ) power fit. The results obtained with the 1SQ and L1 test cases are superimposed in red for contrast. Both test data are in good agreement with the results presented by Bees et al [2], where the 1SQ data follows the non-proportional fitting and the L1 test data lies closer to the in-phase fitting curve. A similar result is illustrated in Figure 5, in the stress-life comparison. All test conditions with $F=1$, i.e. with a phase shift of $90^{\circ}$, presented similar fatigue lives for equivalent stress levels. In contrast, the L1 test case, which presents $F=0.44$, gave considerably longer fatigue lives, comparable to the results obtained by Bees [8] for in-phase loading. Figure 5 also suggest that for stress levels near the yield strength of RR1000, only a minor cross-hardening effect is observed, as the cross, CX1 and CX2, and the HG load paths gave similar fatigue lives. The same is observed in the comparison between 4SQ and 1SQ test cases.

Table 2 - Normalised stress amplitudes, based on load cell readings, and total fatigue lives.

\begin{tabular}{lcccc}
\multirow{2}{*}{ Load Path } & \multicolumn{3}{c}{ Norm. Stress Amplitudes } & Fatigue life \\
& $\sigma_{y y, a}$ & $\tau_{x y, 0 a}$ & $\sigma_{\mathrm{vM}, \mathrm{a}}$ & $2 N_{f}$ \\
\hline \multirow{3}{*}{ One Square- 1SQ } & 0.639 & 0.329 & 0.856 & 12,903 \\
& 0.634 & 0.372 & 0.904 & 7,625 \\
& 0.680 & 0.393 & 0.962 & 4,992 \\
Four Squares - 4SQ & 0.680 & 0.399 & 0.969 & 4,756 \\
& 0.635 & 0.364 & 0.895 & 9,269 \\
Two Triangles L1 & 0.680 & 0.389 & 0.957 & 4,398 \\
& 0.598 & 0.346 & 0.847 & 52,302 \\
Hour Glass- HG & 0.633 & 0.368 & 0.899 & 24,298 \\
& 0.678 & 0.389 & 0.956 & 13,293 \\
Cross- CX1 & 0.599 & 0.347 & 0.848 & 15,080 \\
& 0.630 & 0.365 & 0.893 & 8,366 \\
Cross- CX2 & 0.676 & 0.391 & 0.958 & 4,752 \\
& 0.629 & 0.365 & 0.892 & 10,596 \\
& 0.675 & 0.385 & 0.949 & 5,432 \\
& 0.630 & 0.366 & 0.893 & 9,094 \\
& 0.676 & 0.387 & 0.952 & 5,151
\end{tabular}

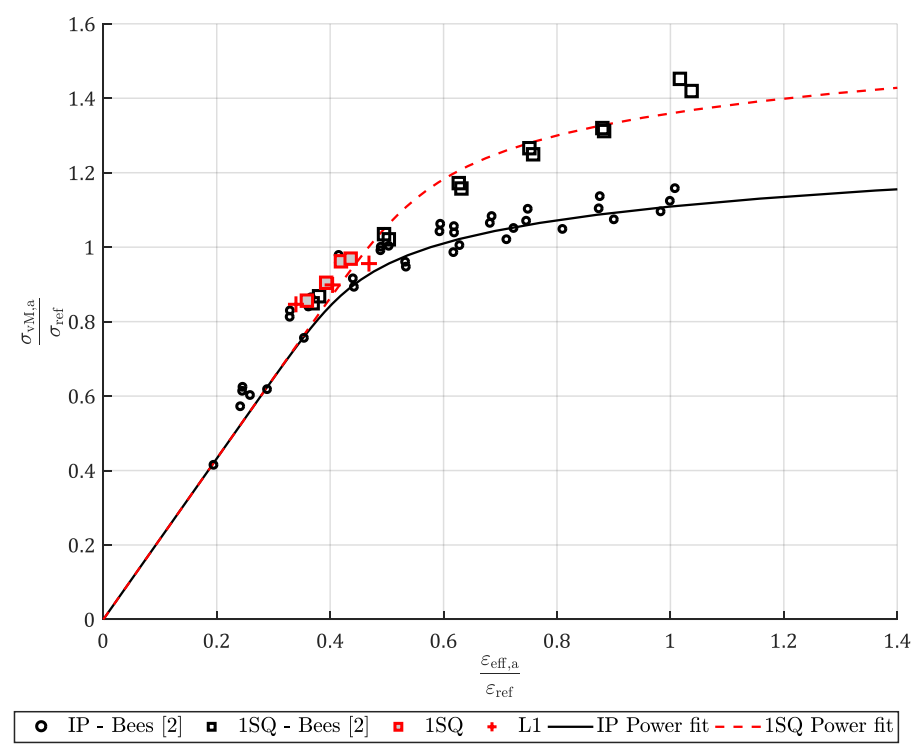

Figure 4 - Cyclic stress-strain response for RR1000 adapted from Bees et al [2] with current test data. 


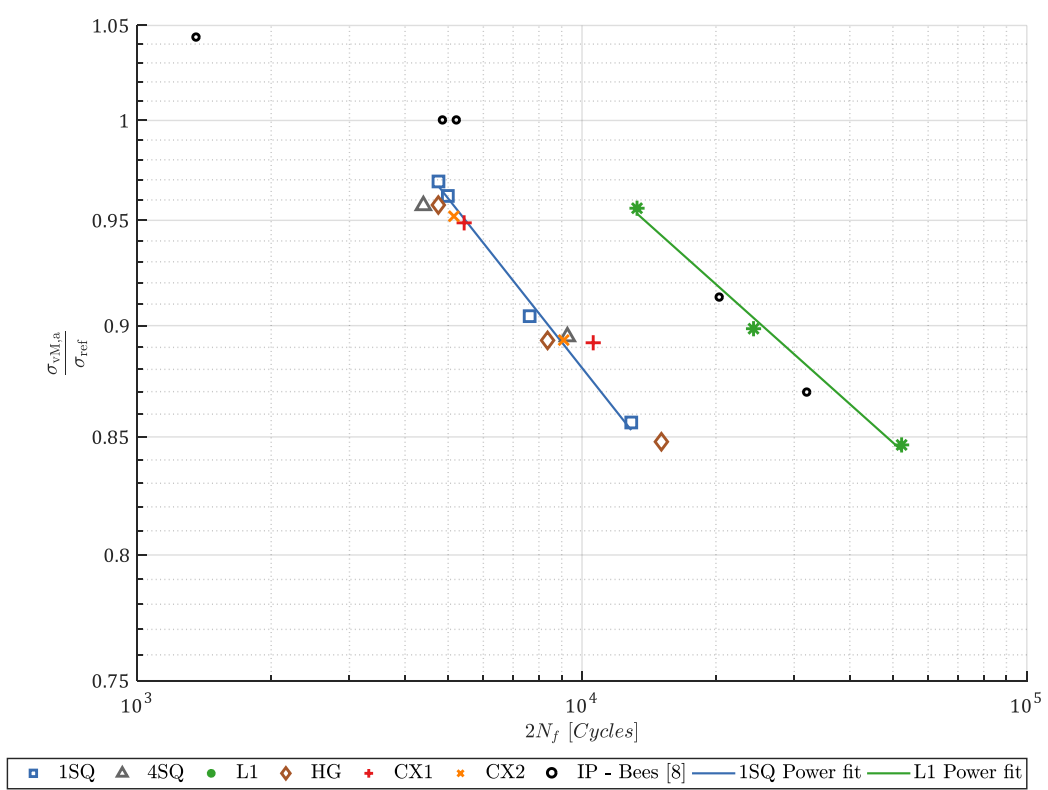

Figure 5 - Stress-life comparison with in-phase test data adapted from Bees [8].

\section{Conclusions}

The non-proportional deformation and fatigue behaviour of RR1000 was investigated under a range of load paths. Results indicate that RR1000 presents no path dependency for stress levels near the yield strength of the material. On the other hand, fatigue lives and cyclic hardening are highly dependent on the degree of non-proportionality. Five of the six load paths investigated have the same degree of load non-proportionality, characterised by the LPD factor $F=1$. A clear distinction is seen between these test cases and test case L1, which presents a lower factor, $F=0.44$. The $\mathrm{L} 1$ test data presented fatigue lives around three times longer that test cases with the higher LPD factor, and strain levels and fatigue lives comparable to in-phase test data.

\section{Acknowledgments}

The authors are grateful for the support of Rolls-Royce plc and the Brazilian National Council of Technological and Scientific Development (CNPq). A portion of this work was part of a Collaborative R\&T Project SILOET supported by the Technology Strategy Board. 


\section{References}

[1] D. Socie and G. Marquis, Multiaxial Fatigue. Warrendale, PA: SAE International, 1999.

[2] M. R. Bees, S. J. Pattison, N. Fox, and M. T. Whittaker, "The non-proportional behaviour of a nickel-based superalloy at room temperature, and characterisation of the additional hardening response by a modified cyclic hardening curve," Int. J. Fatigue, vol. 67, pp. 134-141, 2014.

[3] A. Fatemi and N. Shamsaei, "Multiaxial fatigue: An overview and some approximation models for life estimation," Int. J. Fatigue, vol. 33, no. 8, pp. 948958, 2011.

[4] K. Kanazawa, K. J. Miller, and M. W. Brown, "Cyclic deformation of 1\% Cr-Mo-V steel under out-of-phase loads," Fatigue Fract. Eng. Mater. Struct., vol. 2, no. 2, pp. 217-228, 1979.

[5] N. Cumpsty, Jet Propulsion. Cambridge: Cambridge University Press, 2003.

[6] R. C. Reed, The Superalloys: Fundamentals and Applications. Cambridge University Press, 2008.

[7] S. J. Pattison, "Multi-axial and thermo-mechanical loading effects in Nickel-based disc superalloys," Swansea University, 2012.

[8] M. R. Bees, "The effect of loading proportionality on fatigue life," Swansea University, 2016.

[9] S. H. Doong and D. F. Socie, "Constitutive Modeling of Metals Under Nonproportional Cyclic Loading," J. Eng. Mater. Technol., vol. 113, no. 1, p. 23, 2008.

[10] M. Preuss, P. J. Withers, and G. J. Baxter, "A comparison of inertia friction welds in three nickel base superalloys," vol. 437, pp. 38-45, 2006.

[11] M. W. Brown, "Torsional stresses in tubular specimens," J. Strain Anal. Eng. Des., vol. 13, no. 1, pp. 23-28, 1978. 